\title{
An Effective Rate Distortion Optimization Method for Reliability HEVC Systems
}

\author{
Jinchao Zhao*, Kunqiang Huang, Qiuwen Zhang \\ College of Computer and Communication Engineering, Zhengzhou University of Light Industry, Zhengzhou 450002, China
}

\begin{abstract}
High Efficiency Video Coding (HEVC) possesses a special intact coding flow frame that involves four basic procedures: prediction, transformation, quantization and entropy coding. The whole process has high computational complexity, especially the transformation and quantization, which obstruct HEVC for real-time application. To reduce the computational complexity, computing time, or resources, a fast rate-distortion optimized algorithm for HEVC is presented. The rate distortion optimized transformation (RDOT) with reclassification and the rate-distortion optimized quantization (RDOQ) extensional bypass decision method are employed to save the computational complexity. The RDOT performs initial classification and reclassification for each class to simplify the decorrelation of residual data transformation. Moreover, the RDOQ bypass could reduce the computational complexity by skipping the unnecessary candidates of transformation blocks with high computation. Experimental results reveal that the proposed algorithm implemented reduces HEVC coding complexity and achieves almost the same coding performance.
\end{abstract}

Keywords: High Efficiency Video Coding (HEVC); rate-distortion; optimization

(Submitted on July 25, 2017; Revised on August 30, 2017; Accepted on September 15, 2017)

(This paper was presented at the Third International Symposium on System and Software Reliability.)

(C) 2017 Totem Publisher, Inc. All rights reserved.

\section{Introduction}

The video coding standard has rapidly developed in recent years. With the development of high definition video, the bit-rate becomes one of the most important constraints. It should be as little as possible, which is a very difficult issue. Therefore, a high efficiency standard had been designed recently, which is called High Efficiency Video Coding (HEVC) [10]. This new HEVC standard has especially high performance in practical. For all the new coding tools, rate-distortion optimized (RDO) [9] cost calculation has an important effect on HEVC standard. However, RDO mode decision has huge computational complexity. Low-complexity method of the algorithm is needed in coding process while high computation complexity seriously limits coding efficiency. Moreover, a lower complexity algorithm for transformation and quantization of RDO is of great importance. In this paper, rate-distortion optimized transforms (RDOT) and rate-distortion optimized quantization (RDOQ) are introduced and achieve complexity reduction of HEVC coding processes.

At first, a series of video images with correlation is served and then a set of decorrelation data is obtained. This conventional transformation uses the differences between the current and referenced frames in the form of several parameters. In this paper, we designed an efficient blocks coding method for video frames compression to improve performance. The $\ell_{0}$ norm is introduced to satisfy the non-zero coefficients with the limited bit-rate. The sparse orthonormal transformation defined as $G_{i}$ classify the class and update the $S_{i}$ iteratively in a loop. After this process, the final transformation coefficients are generated to represent the pixels of image. The results obtained from the process of transformation then experience the rate-distortion optimized quantization bypass procedure to decide whether to utilize RDOQ [7] or not. The following experiment helps judge the quantized levels. If the quantized level is lower than these cases, the RDOQ is meaningless. This quantization method is a choice for the coefficients, which utilizes the RDOQ or the

\footnotetext{
* Corresponding author.

E-mail address: zhaojinchao101@163.com
} 
uniform scalar quantization to be quantized.

This paper is organized in the following. Section 2 shows work about RDO. Section 3 introduces our proposed algorithm including rate-distortion optimized transforms and the rate-distortion optimized quantization decision method. The experimental results comparisons are explained in Section 4. At last, this paper is summarized in Section 5.

\section{Related Work}

Recently, several optimized algorithms have been proposed about the RDO method on transformation and quantization. Arrufat et al. proposed a method in [1] about a cost calculation that forms the constraint condition for bit-rate by new RDOTs method to reduce BD-rate [5] of the HEVC. Sezer et al. proposed a method in [14] that the block-based transform optimization and corresponding video frames compression method classify image information and transform coefficients information. Lee et al. proposed a method in [8] that the RDOQ decision selects the transform blocks, which give less or no coding gain to skip to reduce the complexity of quantization. Stankowski et al. proposed an algorithm in [13] that as the exact cost estimation results in variety with RDOQ compared to HEVC Test Model when improving the compression ratios of the RDOQ. Zhang et al. proposed a new point of RDO in [17] that it transforms the DCT coefficients by the removal algorithm and all-zero block-based RDOQ skip algorithm to save the RDOQ time and improve the encoding efficiency. Zou et al. proposed a new rate-distortion optimized transformation method in [16] by training transformations and selecting the applicable transformation pattern to achieve BD rate reduction under the Intra Main.

Admittedly, these methods above have all proven to improve the performance of the HEVC significantly in terms of reducing BD-rate or improving compression ratios to save time and improve efficiency. However, these algorithms improve the bit rate under the complicated constraint of conventional training transformation, rather than simplify the constraint condition, and the process of quantization is only sped up by the variable of cost estimation or the compression ratios of removal DCT coefficients or all-zero skip methods. Therefore, it is necessary to combine the methods together to simplify the constraint and accelerate the block skip decision to improve the performance of both transformation and quantization process.

\section{Effective rate distortion optimization method}

\subsection{Rate-Distortion Optimized Transforms}

The transformation process in image processing can seem as the course of decorrelation, which generates the frame residuals data. And, the features of the image can be extracted by utilizing the properties of residuals data transmission in the form of several parameters. Furthermore, the original HEVC encoders cannot meet the requirements of a lower computation complexity, which the residuals of transmission channel would be encoded as minimum resource efficiency. The feature of the video encoder is corresponding to the bit compression that is experienced with RDO calculation process. Due to the difficult coding process of simulating a practical HEVC coder, a replaced $\ell_{0}$ parameter pattern has been proposed to satisfy the bit compression and transmission condition for the residuals.

In this paper, a transform design that takes advantage of the invertible orthonormal transform is introduced. The sparse orthonormal transforms are defined as $G_{i}(N \times N)$ with $k$ class $(i=1, \ldots, k)$. After designing an initial classification for each class, the blocks re-classification process is performed by utilizing the $\ell_{0}$ norm parameter when the classification and associated transforms are both optimal. This is reasonable since proposed method can be seen as the optimized transmission process due to the fact that the results of nonlinear simulative transform coding are similar to rate distortion optimized results. And, the replaced method utilizing directional features to classify the coding blocks firstly. We can get amazing optimized results of structure-preserving and parameter classification that are like those of most researchers. The minimized transmission function is shown as below for the training data:

$$
\min _{\mathrm{G}_{\mathrm{i}}} \sum_{j \in S_{i}}\left\{\min _{c_{i}^{j}}\left[\left\|x^{j}-G_{i} c_{i}^{j}\right\|^{2}+\lambda\left\|c_{i}^{j}\right\|_{0}\right] \text { s.t. } \boldsymbol{G}_{\boldsymbol{i}}^{\boldsymbol{T}} \boldsymbol{G}_{\boldsymbol{i}}=\boldsymbol{I}\right.
$$


The parameter $S_{\mathrm{i}}$ represents a $i$ groups blocks extracted from coding block. $\mathrm{x}^{j}(N \times 1)$ represents the depth of blocks. $G_{i}$ denotes the process of orthonormal transforms optimization. $c_{i}^{j}(N \times 1)$ denotes the coefficients for $\mathrm{x}^{j}$. And, the introduced $\ell_{0}$ norm has the same amounts of nonzero data in $c_{i}^{j}$ that is represented by the aforementioned functions in (1).

The optimization method is proposed to compute $S_{i}$ and $G_{i}$ in a loop. Firstly, the training set is classified by utilizing gradient features to achieve the first class and calculate initial $S_{i}$ of the blocks. Then, the orthogonality process $G_{i}$ is updated with the K-L Transforms based on the property of blocks correlation related to classified groups that are impacted by $S_{i}$. Finally, utilizing the given classification method, we can perform the optimal transformation pattern. The blocks would be reclassified according to the following equation:

$$
\operatorname{Lable}\left\{\mathrm{x}^{j}\right\}=\arg \min _{i}\left[\min _{c_{i}^{j}}\left[\left\|\mathrm{x}^{j}-G_{i} c_{i}^{j}\right\|^{2}+\lambda\left\|c_{i}^{j}\right\|_{0}\right]\right]
$$

This function can get the new $S_{i}$. And the optimization process would perform constantly until convergence.

Based on the proposed optimization method, the first procedure is to calculate the optimal coefficients for the transformation $G_{i}$. The sparse representation for the function is as follows:

$$
\mathrm{c}_{i}^{j}=\arg \min _{d}\left(\left\|\mathrm{x}^{j}-G_{i} d\right\|^{2}+\lambda\|d\|_{0}\right)
$$

Then, the results of above function can be considered as a threshold for the elements of $\mathrm{v}_{i}^{j}=G_{i}^{T} \mathrm{x}^{j}$ under the condition of the threshold is set as $\sqrt{\lambda}$. It can be represented as follow:

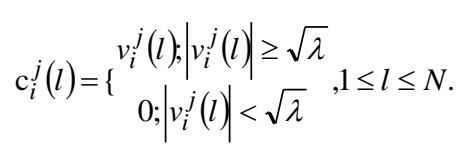

Second, perform the optimal orthonormal transformations. This procedure can reduce the reconstruction difference of the obtained coefficients to a minimum.

$$
G_{i}=\arg \min _{H}\left(\sum_{j \in S_{i}}\left\|x^{j}-H c_{i}^{j}\right\|^{2}\right)
$$

The function can be simplified as:

$$
\min _{H} \sum_{j \in S_{i}}\left[\operatorname{Tr}\left(\left(x^{j}-H c_{i}^{j}\right)^{T}\left(x^{j}-H c_{i}^{j}\right)\right)\right]
$$

The is the trace of matrix. Define $Y=\sum_{j \in S_{i}} c_{i}^{j} x^{j T}$ and set $U \Lambda^{1 / 2} V^{T}$ as SVD for Y [11]. $U, V$ are orthonormal and $\Lambda$ is diagonal. And, let $P=V^{T} H U$ with $P, V, H, U$ all orthonormal.

Assume after a series of simplifying process, when $P=I$ with $P^{T} P=I$, the $G_{i}$ of function (1) can be expressed as: 


$$
G_{i}=V U^{T}
$$

We can utilize the results of (3) and (5) to solve the (1).

Finally, the last step is the circle process of utilizing the reclassified data. After the above optimization process, all the blocks would be reclassified by taking the advantage of determined transforms utilizing already classified blocks until the loop convergence. This step is performed based on updated and new sets of transformations.

\subsection{Rate-Distortion Optimized Quantization Decision}

The rate-distortion optimized quantization (RDOQ) method brings obvious coding performance improvement. However, this conventional quantization process introduces large computation complexity. The RDOQ performs RD cost estimation based on the following method we proposed for all candidates of all coefficients to select the blocks, which could save some unnecessary computation complexity by taking advantage of utilizing the quantized levels [12]. The proposed method extends the RDOQ through developing a RDOQ decision method. And, the decision algorithm determines the TBs that utilize the uniform scalar quantization [15] to be quantized. With the RDOQ bypass decision method, large computation complexity is saved.

The RDOQ has a particular advantage of coding gain that possesses several quantized levels for coefficients in TBs. However, when the RDOQ satisfies the following two cases, it is meaningless to the coding performance.

- The parameters are zero.

- Quantization levels are similar to uniform scalar results.

Table 1 indicates the statistical possibility for quantization results of transform blocks where the experienced conventional algorithm $\left(G_{R D O Q}\right)$ is similar to uniform scalar process $\left(G_{S Q}\right)$. And, $G_{0}$ is the first special occasion that parameters in blocks are approximatively equal to zero. Table 1 shows that the parameters of TB are all quantized to zero under the common condition of $G_{S Q}=G_{R D O Q}$. Therefore, it can deduce that the coding block would be considered as skip blocks in the RDOQ process if the magnitude of transmission parameters is less than the existing quantization level. Based on above analysis, the quantization procedure with large calculation would be omitted. Furthermore, there also exists the second occasion of $G_{S Q}=G_{R D O Q}$ since the level is nonzero. And, the average step size values with different block sizes in RDOQ is represented in Table 2. It means that the process of RDOQ would not be significant if the quantization levels are lower than the average value 2.2. This is due to the quantization levels in aforementioned occasions of which most are capable of setting as zero. And, even the nonzero levels are sufficiently low since it should be approximated to an integer. The following experimental results show that if the quantized levels are satisfied with both cases, the performance improvement of RDOQ would be limited without considering its large computation complexity. This contributes to RDOQ simplified bypass decision method. In the Table 2 , the formula can summarize the $G_{S Q}=G_{R D O Q}$ as follow:

$$
\left.\sum_{m=0}^{N-1} l_{m}=\sum_{m=0}^{N-1} \mid \frac{\left|c_{m}\right|}{Q_{s}}+f\right\rfloor \cong \sum_{m=0}^{N-1} \frac{\left|c_{m}\right|}{Q_{s}}=\frac{1}{Q_{s}} \sum_{m=0}^{N-1}\left|c_{m}\right|
$$

Where $l_{m}$ represents $\mathrm{m}$-th level and $\mathrm{c}_{m}$ represents unquantized coefficient. And, $N$ represents the number of coefficients, $f$ denotes the offset parameter of scalar quantization process. Moreover, this RD simplification procedure is unnecessary in the case of $\sum_{i=0}^{N-1} l_{m}$ less than threshold $T$, which is explained by the following equation:

$$
\sum_{m=0}^{N-1} l_{m}<T \rightarrow \sum_{m=0}^{N-1} l_{m} \cong \frac{1}{Q_{s}} \sum_{i=0}^{N-1}\left|c_{m}\right|<T
$$

Finally, the equation could be simplified as the following simple decision condition (10).

$$
\sum_{m=0}^{N-1}\left|c_{m}\right|<T \cdot Q_{s}
$$


Based on above inequality, if the coefficients satisfy the condition, the optimization process would utilize the uniform scalar quantization. Else, performing the RDOQ method. In the proposed decision process, set 2.2 that is calculated in Table 2 as threshold $T$. In Tables 1 and 2, several sequences (Kimono, Keiba, BasketballPass, ChinaSpeed, and BasketballDrillText) are utilized. Especially, the series of Basketball sequences are selected to represent the properties of some other test sequences.

\begin{tabular}{|c|c|c|c|c|}
\hline \multirow{3}{*}{$\begin{array}{c}\text { TB size } \\
4 \times 4\end{array}$} & \multicolumn{2}{|c|}{ Case $A: G_{\mathrm{SQ}}=\mathbf{G}_{\mathrm{RDOQ}}$} & \multicolumn{2}{|c|}{ Case B: $\mathbf{G}_{\mathrm{SQ}} \neq \mathbf{G}_{\mathrm{RDOQ}}$} \\
\hline & $\mathbf{P}(\mathbf{A})$ & $\mathbf{P}=\left(\mathbf{G}_{\mathrm{RDOQ}}=\mathbf{G}_{0} \mid \mathbf{A}\right)$ & $\mathbf{P}(\mathbf{B})$ & $\left.{ }_{O Q}=\mathbf{G}_{0} \mid \mathbf{B}\right)$ \\
\hline & 0.27 & 0.651 & 0.73 & 0.235 \\
\hline $8 \times 8$ & 0.20 & 0.654 & 0.80 & 0.215 \\
\hline $16 \times 16$ & 0.09 & 0.798 & 0.91 & 0.232 \\
\hline $32 \times 32$ & 0.01 & 0.883 & 0.99 & 0.172 \\
\hline Avg. & 0.14 & 0.747 & 0.86 & 0.213 \\
\hline
\end{tabular}

\begin{tabular}{|c|c|c|}
\multicolumn{3}{|c|}{ Table 2. Sum of quantized levels after RDOQ } \\
\hline TB size & $\begin{array}{c}\mathbf{G}_{\mathbf{S Q}}=\mathbf{G}_{\text {RDOQ, }} \\
\mathbf{G}_{\text {RDOQ }} \neq \mathbf{G}_{\mathbf{0}}\end{array}$ & $\begin{array}{c}\mathbf{G}_{\text {SQ }} \neq \mathbf{G}_{\text {RDOQ, }} \\
\mathbf{G}_{\text {RDOQ }} \neq \mathbf{G}_{\mathbf{0}}\end{array}$ \\
\hline $4 \times 4$ & 1.95 & 10.11 \\
\hline $8 \times 8$ & 1.58 & 18.38 \\
\hline $16 \times 16$ & 1.81 & 53.43 \\
\hline $32 \times 32$ & 3.56 & 213.43 \\
\hline Avg. & 2.23 & 73.84 \\
\hline
\end{tabular}

\section{Experimental results analysis}

The RD performance of transformation process is evaluated by the Bjøntegaard Distortion (BD) rate in this paper. In Table 3 , most video sequences have large motion regions, which include small sizes of $4 \times 4$ and $8 \times 8$. Based on this feature, these small size coding units are selected as the set of train data. Small sizes also have a balance between the performance and calculated quantity. In order to maintain system stability among different video sequences resolution ratio, a mass of prediction residuals from numbers blocks should be trained. Moreover, the different quantization parameters (QPs) and resolutions under the common test conditions [2] should be considered.

Table 3. AI HEVC distribution and Y BD-Rate saving referred to HEVC

\begin{tabular}{|c|c|c|c|c|c|c|c|}
\hline & Sequence & $\begin{array}{c}\text { Surfac } \\
4 \times 4\end{array}$ & $\begin{array}{r}\text { by blo } \\
8 \times 8\end{array}$ & $\begin{array}{l}\text { sizes } \\
16 \times\end{array}$ & $\begin{array}{c}E V C)(\%) \\
32 \times 32\end{array}$ & $\begin{array}{c}\text { Y BI } \\
\text { redu } \\
\text { AI }\end{array}$ & $\begin{array}{r}\text {-rate } \\
\text { ce(\%) } \\
\text { RA }\end{array}$ \\
\hline \multirow{5}{*}{$\begin{array}{c}\text { Class A } \\
(2560 \times \\
1600)\end{array}$} & PeopleOnStreet & 18 & 30 & 33 & 19 & 1.66 & 1.80 \\
\hline & Traffic & 14 & 25 & 33 & 28 & 1.66 & 1.80 \\
\hline & NebutaFestival & 3 & 7 & 20 & 70 & 0.18 & 0.05 \\
\hline & SteamLocomotiveTran & 1 & 7 & 22 & 70 & 0.00 & -0.51 \\
\hline & Average & 9 & 17 & 27 & 47 & 1.28 & 0.34 \\
\hline \multirow{6}{*}{$\begin{array}{c}\text { Class B } \\
(1920 \times \\
1080)\end{array}$} & BasketballDrive & 8 & 22 & 29 & 41 & 1.04 & 0.07 \\
\hline & BQTerrace & 14 & 40 & 22 & 24 & 1.61 & 0.94 \\
\hline & Cactus & 16 & 23 & 31 & 30 & 1.85 & 1.38 \\
\hline & Kimono1 & 3 & 9 & 23 & 65 & 0.30 & 0.24 \\
\hline & ParkScene & 17 & 23 & 29 & 31 & 1.28 & 1.19 \\
\hline & Average & 12 & 23 & 27 & 38 & 1.22 & 0.76 \\
\hline \multirow{5}{*}{$\begin{array}{c}\text { Class C } \\
(832 \\
\times \\
480)\end{array}$} & BasketballDrill & 29 & 33 & 25 & 13 & 2.76 & 2.63 \\
\hline & BQMall & 24 & 31 & 27 & 18 & 1.83 & 1.28 \\
\hline & PartyScene & 47 & 35 & 16 & 3 & 3.06 & 2.14 \\
\hline & RaceHorses & 18 & 24 & 30 & 28 & 1.87 & 0.80 \\
\hline & Average & 21 & 27 & 28 & 24 & 2.38 & 1.72 \\
\hline \multirow{5}{*}{$\begin{array}{c}\text { Class D } \\
(416 \\
\times \\
240)\end{array}$} & BasketballPass & 22 & 26 & 28 & 24 & 2.33 & 0.98 \\
\hline & BQSquare & 47 & 25 & 18 & 10 & 2.80 & 1.94 \\
\hline & BlowingBubbles & 50 & 34 & 14 & 2 & 3.38 & 2.14 \\
\hline & RaceHorses & 26 & 31 & 27 & 16 & 2.23 & 0.96 \\
\hline & Average & 36 & 29 & 22 & 13 & 2.69 & 1.51 \\
\hline
\end{tabular}

In the designed process, two RDOT systems would correspond to a single and a multiple transform. Both systems have numbers of extra RDOTs to test data. The encoder is designed to perform the RDO iteration process, and the transformation 
system will choose the most adaptive transformation pattern and code it as bit steam. The training is performed under the condition of all intra (AI) common test configuration [3] for intra prediction residuals extracted from video sequences. This process also tested the condition of random access (RA).

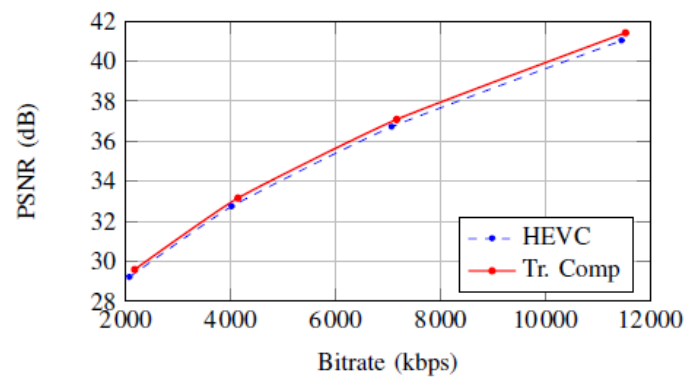

Figure 1. BD-rate saving for Blowing Bubbles:-3.38\%

An experiment of BD-rate curve is shown in Figure 1, in which PSNR is increasing with bitrate improvement. Moreover, all QPs in the performance improvement process are apparent and the rates also improved consistently through measurement by by the Peak Signal Noise Ratio (PSNR) [5]. Though improvement is inconspicuous in visual, the objective quality improvement in the decoder is realized.

Table 3 reveals the blocks size distribution for the test video sequences with different resolutions under the condition of AI. The results for BD rate improvements in the table show that it outperforms HEVC original test mode in terms of quality promotion. This promotion covers all selected sequences, especially the low resolution. The improvement under the condition of AI and RA realized about $2 \%$ and $1 \%$, respectively. The performance of blocks coding also has large improvement on RA test configuration, which achieves high-quality video coding.

From the Table 3, the improvement is highly dependent on the test blocks $(4 \times 4$ and $8 \times 8)$ for HEVC test mode. Therefore, an improvement on test sequences is high if it has large sample distribution for $4 \times 4$ or $8 \times 8$ size blocks. On the other side, the sequences with higher resolution have less or even loss. This is because higher resolution sequences have small distribution of coding blocks. Based on above analysis, the most important feature for system performance is block size.

Table 4. Signaling factor for Y BD-Rate

\begin{tabular}{|c|c|c|c|}
\hline \multicolumn{2}{|c|}{ Configuration } & \multicolumn{2}{|c|}{ Y BD-rate(\%) } \\
\hline $4 \times 4$ & $8 \times 8$ & No Signaling & Signaling \\
\hline $1+4$ & ----- & -8.42 & -1.40 \\
\hline $\begin{array}{ll}---- \\
-\end{array}$ & $1+16$ & -7.01 & -2.46 \\
\hline $1+4$ & $1+16$ & -11.27 & -3.38 \\
\hline
\end{tabular}

Table 5. Test sequences for experiment

\begin{tabular}{|c|c|c|c|c|}
\hline Class & Sequence & Resolution & Frame Rate(HZ) & Frames encoded \\
\hline A & $\begin{array}{c}\text { Traffic } \\
\text { PeopleOnStreet } \\
\text { PartScene }\end{array}$ & $2560 \times 1600$ & $\begin{array}{l}30 \\
30 \\
24\end{array}$ & $\begin{array}{l}300 \\
300 \\
240\end{array}$ \\
\hline B & $\begin{array}{c}\text { BQTerrace } \\
\text { BasketballDrive } \\
\text { BasketballDrill } \\
\end{array}$ & $1920 \times 1080$ & $\begin{array}{l}60 \\
50 \\
50 \\
\end{array}$ & $\begin{array}{l}600 \\
500 \\
500 \\
\end{array}$ \\
\hline $\mathrm{C}$ & $\begin{array}{c}\text { BQMall } \\
\text { PartyScene } \\
\text { BasketballPass }\end{array}$ & $832 \times 480$ & $\begin{array}{l}60 \\
50 \\
50\end{array}$ & $\begin{array}{l}600 \\
500 \\
500\end{array}$ \\
\hline $\mathrm{D}$ & $\begin{array}{c}\text { RaceHorses } \\
\text { BlowingBubbles } \\
\text { FourPeople }\end{array}$ & $416 \times 240$ & $\begin{array}{l}30 \\
50 \\
60 \\
\end{array}$ & $\begin{array}{l}300 \\
500 \\
600 \\
\end{array}$ \\
\hline $\mathrm{E}$ & $\begin{array}{c}\text { Johnny } \\
\text { KristenAndSara } \\
\text { BasketballDrillText }\end{array}$ & $\begin{array}{l}1280 \times 720 \\
832 \times 480\end{array}$ & $\begin{array}{l}60 \\
60 \\
50 \\
\end{array}$ & $\begin{array}{l}600 \\
600 \\
500 \\
\end{array}$ \\
\hline $\mathrm{F}$ & $\begin{array}{c}\text { SlideShow } \\
\text { SlideEditing }\end{array}$ & $1280 \times 720$ & $\begin{array}{l}20 \\
30\end{array}$ & $\begin{array}{l}500 \\
300\end{array}$ \\
\hline
\end{tabular}

In the Table 4, the signal transmission pattern has an effect on the performance of designed system. Based on the BlowingBubbles coding process, signaling $4 \times 4$ blocks requires greater performance loss compared with the larger blocks. Therefore, the percentages of improvement reduction of residual coding and signaling are greater for small size blocks. 
Finally, utilizing the advantages of spatial redundancies, signal transmission system performance loss would be reduced. According to the results of the experimental sample, the proposed design scheme can be applied to other blocks transmission processes of RDOT. As the new quadtree partitioning structure of HEVC, the complexity per block may increase for a specific case. Because of the different combinations of blocks sizes, the designed system cannot test all the candidates. However, the time adds a little based on (1) due to non-separable transformation. The decoder can copy the information of the encoder directly by utilizing the proposed system.

The computation complexity reduction of the RDOQ decision process is proven to be effective in HM 11.0 test model environment [6]. Experiment is performed under the condition of AI and RA and low delay (LD-main) same with [4]. All test sequences are shown in Table 5 with QP equals to 22, 27, 32 and 37, respectively. The BDBR [5] evaluates the performance improvement against the Anchor method. HM 11.0 experiment mode with RDOQ=ON. The average saving time (AST) showing as follows:

$$
\operatorname{AST}(\%)=\frac{T_{A}-T_{P}}{T_{A}} \times 100
$$

Where $T_{A}$ is Anchor coding time and $T_{P}$ is our fast decision algorithm coding cost in RDOQ process. The higher PSNR reveals that the decision algorithm outperforms the contrastive method. And, the higher AST indicates better performance in terms of time saving for our fast decision algorithm.

Table 6. Results of the RDOQ decision compared with anchor

\begin{tabular}{|c|c|c|c|c|c|c|c|c|c|}
\hline \multirow{2}{*}{ Sequence } & \multicolumn{3}{|c|}{ AI-Main } & \multicolumn{3}{|c|}{ RA-main } & \multicolumn{3}{|c|}{ LD-main } \\
\hline & BDBR & PSNR & AST & BDBR & PSNR & AST & BDBR & PSNR & AST \\
\hline Traffic & 0.0 & 0.000 & 10.5 & 0.0 & 0.002 & 9.1 & -- & -- & -- \\
\hline PeopleOnStreet & 0.0 & 0.000 & 9.8 & 0.0 & 0.000 & 10.2 & -- & -- & -- \\
\hline ParkScene & 0.0 & 0.000 & 11.1 & 0.0 & 0.001 & 9.4 & 0.0 & 0.001 & 9.0 \\
\hline BQTerrace & 0.0 & -0.001 & 10.7 & 0.1 & -0.002 & 9.8 & -0.1 & 0.001 & 8.9 \\
\hline BasketballDrive & 0.0 & 0.000 & 8.9 & -0.1 & 0.001 & 12.4 & 0.0 & 0.002 & 9.3 \\
\hline BaskeballDrill & 0.0 & 0.000 & 6.9 & 0.2 & -0.008 & 11.2 & 0.0 & 0.000 & 8.7 \\
\hline BQMall & 0.0 & -0.001 & 10.0 & 0.1 & -0.006 & 9.5 & 0.1 & -0.005 & 8.0 \\
\hline PartyScene & 0.1 & -0.006 & 10.7 & 0.2 & -0.011 & 8.9 & 0.2 & -0.010 & 9.7 \\
\hline BasketballPass & 0.0 & 0.002 & 12.4 & 0.2 & -0.009 & 8.4 & 0.2 & -0.009 & 8.4 \\
\hline RaceHorses & 0.0 & 0.000 & 12.9 & 0.2 & -0.011 & 9.4 & 0.2 & -0.011 & 9.8 \\
\hline BlowingBubbls & 0.0 & -0.001 & 12.6 & 0.0 & 0.000 & 11.3 & 0.0 & 0.000 & 10.8 \\
\hline FourPeople & 0.0 & -0.001 & 10.9 & 0.0 & 0.002 & 11.3 & -0.3 & 0.009 & 10.1 \\
\hline Johnny & 0.0 & 0.000 & 8.5 & 0.1 & 0.000 & 9.6 & -0.5 & 0.007 & 8.2 \\
\hline KristenAndSara & 0.0 & 0.000 & 8.9 & 0.1 & -0.003 & 10.5 & -0.4 & 0.015 & 10.6 \\
\hline BasketballDrillText & 0.0 & -0.001 & 9.8 & 0.1 & -0.002 & 8.7 & 0.2 & -0.007 & 9.3 \\
\hline SlideShow & 0.3 & -0.025 & 25.2 & 0.4 & -0.038 & 29.4 & 0.5 & -0.034 & 26.4 \\
\hline SlideEditing & 0.8 & -0.119 & 9.7 & 0.9 & -0.247 & 11.0 & 0.4 & -0.064 & 12.7 \\
\hline $\begin{array}{c}\text { Only } \\
\text { Training seq. }\end{array}$ & 0.0 & 0.0004 & 11.11 & 0.12 & -0.0059 & 8.59 & 0.19 & -0.0084 & 8.89 \\
\hline $\begin{array}{c}\text { Avg-w/o training } \\
\text { seq. }\end{array}$ & 0.08 & -0.010 & 11.40 & 0.15 & -0.0213 & 11.94 & 0.01 & -0.0069 & 10.94 \\
\hline overall & 0.07 & -0.009 & 11.23 & 0.15 & -0.0195 & 11.19 & 0.04 & -0.0071 & 10.52 \\
\hline
\end{tabular}

Table 6 shows a better quantization efficiency for fast decision algorithm. As the table shows, the proposed decision algorithm achieves large time reduction of $11.2 \%, 11.2 \%$ and $10.5 \%$ under the condition of AI, RA, LD, respectively. Meanwhile, about $0.09 \%$ BDBR reduction and about $0.011 \mathrm{~dB}$ PSNR drop compared with the anchor method, respectively. Therefore, the coding loss is negligible.

Based on the extensive experiment, the proposed method has a better performance compared with conventional HEVC encoder with negligible coding loss, especially under the high-quality coding performance condition. Furthermore, this method could also generate a lookup table for RD cost calculation. The foregoing result analysis indicates that the proposed overall algorithm could keep nearly the same RD performance compared with the original HEVC encoders, while significantly reducing the coding time of the rate distortion optimization process considerably.

\section{Threats}

As the table 3 shown, if our proposed method utilizing the Ultra High Definition(UHD) video sequences to test, the performance improvement is less than lower resolution sequences. Specifically, the result of SteamLocomotiveTrain 
sequence has an unusual BD rate increase about $0.51 \%$ under the condition of RA compare with original HEVC encoder. Because the aforementioned sequence contains small amounts of motion blocks that are partitioned as small size blocks, the texture variation is little. Therefore, the transformation process generates less BD reduction and even increases since the proposed design is correlated to the low percentage of small blocks in test sequence. In our method, less small blocks will limit the performance improvement.

And, from the Table 4, the signal transmission pattern has a profound effect on BD rate reduction. The negative values reveal $\mathrm{BD}$ reduction. In this proposed method, the larger reduction represents the better performance in term of $\mathrm{BD}$ rate. And, the results indicate that the no signaling pattern is efficient if the decoder copies the transformation information directly. Therefore, we should take full use of the signaling pattern; for example, spatial redundancies that transform without any signaling needs further research.

\section{Conclusions}

In this paper, a fast rate distortion optimization algorithm to reduce computational complexity of HEVC in the transformation and quantization process is designed. The proposed transformation design of RDOT and the quantization of RDOQ bypass decision extent to RDOQ perform in succession could improve the performance of HEVC. The transformation classifies the blocks and classes in the training set. Moreover, the RDOQ bypass decision algorithm skips some unnecessary candidates to save the computational complexity. Finally, the comparative experimental results demonstrate that the optimal transformation method outperforms the conventional blocks transformation and achieves apparently compress and the quantization procedure reduces the encoding time apparently with negligible BDBR increase. Therefore, the proposed rate distortion optimization method can significantly reduce the computational complexity of HEVC with almost the same coding efficiency with the original encoders.

\section{Acknowledgements}

The authors would like to thank the editors and anonymous reviewers for their valuable comments. This work was supported in part by the National Natural Science Foundation of China under grant No. 61771432, 61302118, 61401404, and 61502435, the Program for Science and Technology Innovation Talents in Universities of Henan Province under grant No.17HASTIT022, the Funding Scheme of Young Key Teacher of Henan Province Universities under grant No. 2016GGJS-087, the Scientific and Technological Project of Henan Province under grant No.172102210064, the Scientific and Technological of the Education Department of Henan Province under grant No. 17B510011, and in part by the Doctorate Research Funding of Zhengzhou University of Light Industry, under grant No. 2013BSJJ047.

\section{References}

1. A. Arrufat, P. Philippe, and O. Déforges, "Rate Distortion Optimised Transform Competition for Intra Coding in HEVC," in Visual Communications and Image Processing, 2014. IEEE Proceedings on, pp. 73-76, 2014

2. F. Bossen, "Common Test Conditions and Software Reference Configurations," Joint Collaborative Team on Video Coding (JCT-VC), JCTVC-F900, 2011.

3. F. Bossen, "Common Test Conditions and Software Reference Configurations," ITU-T, Geneva, Switzerland, Tech. Rep. JCTVC-I1100, May. 2012

4. F. Bossen, "Common HM Test Conditions and Software Reference Configuration," Joint Collaborative Team on Video Coding, document JCTVC-L1100, Geneva, Switzerland, Jan. 2013.

5. G. Bjøntegaard, "Calculation of Average PSNR Differences Between RD-curves," ITU-T, Austin, Texas, Tech. Rep. VCEGM33, Apr. 2001

6. "High Efficiency Video Coding Test Model Software Version 11," Available at https://hevc.hhi.fraunhofer.de/svn/svn_HEVCSoftware, Last accessed on October 1, 2017

7. M. Karczewicz, Y. Ye, and I. Chong, "Rate Distortion Optimized Quantization," document ITU-T SG16 Q. 6, VCEG-AH21, Antalya, Turkey, Jan. 2008

8. H. Lee, S. Yang, Y. Park, and B. Jeon, "Fast Quantization Method with Simplified Rate-Distortion Optimized Quantization for An HEVC Encoder," IEEE Transactions on Circuits and Systems for Video Technology, Vol. 26, Issue 1, pp. 107 - 116, Jan. 2016

9. A. Ortega, and K. Ranchandra, "Rate-distortion Methods for Image and Video Compression," IEEE Signal Process.Mag., vol. 15, no. 6, pp. 23-50, Nov. 1998.

10. G. J. Sullivan, J. R. Ohm, W. J. Han, and T. Wiegand, "Overview of the High Efficiency Video Coding (HEVC) Standard," IEEE Trans. Circuits Syst. Video Technol., vol. 22, no. 12, pp. 1649-1668, Dec. 2012.

11. G. Strang, L. Algebra and Its Applications, Brooks Cole, 3rd edition, 1998.

12. J. Sole, R. Joshi, N. Nguyen, T. Y. Ji, M. Karczewicz, G. Clare, F. Henry, and A. Duenas "Transform Coefficient Coding in 
HEVC," IEEE Trans. Circuits Syst. Video Technol., vol. 22, no. 12, pp. 1765-1777, Dec. 2012.

13. J. Stankowski, C. Korzeniewski, M. Domanski, and T. Grajek, "Rate-distortion Optimized Quantization in HEVC: Performance Limitations," in Picture Coding Symposium (PCS), IEEE, pp.85-89, July 2015

14. O. G. Sezer, O. Harmanci, and O. G. Guleryuz, "Sparse Orthonormal Transforms for Image Compression," In Proc. of 15th IEEE Int. Conf. on Image Processing, San Diego, CA, pp. 149-152, Oct. 2008

15. Y. Yu, M. Z. Coban, and M. Karczewicz, "Level Decision in Rate Distortion Optimized Quantization,” U.S.Patent 20130272 385 A1, Mar. 2013

16. F. Zou, O. Au, C. Pang, J. Dai, X. Zhang, and L. Fang, "Ratedistortion Optimized Transforms Based on the Lloyd-Type Algorithm for Intra Block Coding," IEEE Journal of Selected Topics in Signal Processing, vol. 7, no. 6, pp. 1072-1083, Dec. 2013

17. Y. Zhang, R.Tian, J. Liu, and N. Wang, "Fast Rate Distortion Optimized Quantization for HEVC," Visual Communications and Image Processing (VCIP), pp.1-4 Dec. 2015 